\title{
Node Clustering Based on Overlapping FoVs for Wireless Multimedia Sensor Networks
}

\author{
Mohammad Alaei, Jose M. Barcelo-Ordinas \\ Computer Architecture Department \\ Universitat Politècnica de Catalunya (UPC) \\ Barcelona, Spain \\ E-mail: \{malaei, joseb $\}$ (at)ac.upc.edu
}

\begin{abstract}
Wireless Multimedia Sensor nodes sense areas that are uncorrelated to the areas covered by radio neighbor sensors. Thus, node clustering for coordinating multimedia sensing and processing cannot be based on classical sensor clustering algorithms. This paper presents a clustering mechanism for Wireless Multimedia Sensor Networks based on overlapped Field of View (FoV) areas. Today, for random deployments, dense networks of low cost, low resolution and low power multimedia nodes are preferred than sparse cases of high cost, high resolution and high power nodes. Overlapping FoVs in dense networks causes wasting power of system because of redundant sensing of area. The main aim of the proposed clustering method is energy conservation and prolonging network lifetime. This aim is achieved through coordination of nodes belonging to the same cluster in assigned tasks, avoiding redundant sensing or processing.
\end{abstract}

Keywords-Wireless Multimedia Sensor Network; Field of View; Clustering; Coordination; Energy Conservation.

\section{INTRODUCTION}

Wireless Sensor Networks (WSN) [1][2], are considered as autonomous and self-organizing systems consisting of a large number of small, inexpensive, battery-powered communication devices deployed throughout a physical space. These networks are mainly to be used for gathering information related to the surrounding environment (e.g., temperature, humidity, light, etc), and for transmission of the gathered data to a base station (i.e., sink), for further processing. In recent times there has been increased interest in video surveillance and environment monitoring applications. Visual information may be captured from the environment using CMOS cameras embedded in wireless sensor nodes. Wireless Multimedia Sensor Networks (WMSN) [3][4], should be able to process in real-time, retrieve or fuse multimedia data.

Energy conservation and maximization of system lifetime is commonly recognized as a key challenge in the design and implementation of WSNs. One of the subjects that have been propounded for enhancement in efficiency of applications associated with WSNs, is node clustering. Clustering in WSN pursues several objectives: (i) network scalability, (ii) energy conservation, (iii) network topology stabilization, (iv) routing overhead minimization, (v) optimized management strategies to prolong battery life and network lifetime and (vi) efficient data aggregation. Most of the time, distance from nodes to cluster-head or radio coverage (i.e., neighborhood) are the main criterions for node clustering in WSNs [5].
Nevertheless, sensing region of multimedia nodes is very different from ordinary nodes in WSNs. Each multimedia node has a Field of View (FoV) and only can capture images from the objects within that region. In traditional WSNs, the sensor nodes collect information about different phenomena around them from the area determined by the sensing range of the node. However, video cameras capture images of objects of a region that are not necessarily in the camera's vicinity. The object covered by the camera can be distant from the camera and the captured images will depend on the relative positions and orientation of the cameras towards the observed object [6][7][8]. Therefore, because of non-coincidence between radio neighborhood and sensed region by multimedia nodes, node clustering and coverage techniques in WSN do not satisfy WMSN requirements.

As a result of developing in low cost, low power, low resolution camera sensors, having a dense network consisting of multimedia sensors has become applicable. This kind of deploying has more performance than sparse networks of high power, high resolution cameras. However, overlapping FoVs in dense deployments yield wasting power of system because of redundant sensing of area [7]. In this paper, we present an approach for multimedia node clustering that satisfies FoV constraints to solve this problem. In this approach, the overlapping area between FoV of multimedia nodes is the main criterion for node clustering in contrast to radio or distance neighborhood. If the overlapped area between FoV of two nodes is relatively wide, they act similarly from the coverage point of view and we select them as members of a cluster. Thus, nodes belonging to the same cluster may not necessarily be neighbor while have some intersection in covering the sensing area. The main aim of this clustering method is energy conservation and balancing energy level in nodes to prolong network lifetime through coordination of nodes belonging to same cluster and avoiding redundant sensing and processing.

The main contributions of this paper are:

- A node clustering algorithm based on overlapping camera FoVs. Finding the intersection polygons and in particular computing the overlapped areas is the key issue to establish clusters and determine cluster membership.

- Applying coordination among cluster members to conserve energy in them with respect to un-clustered solutions. 
The remainder of this paper is organized as follows: Section II presents related work. Section III describes the way of finding and calculus of overlapping polygons and then proceeds to explain the clustering algorithm. Test results of the algorithm are showed by section IV. Development in energy conservation resulted from clustering is discussed in section V. Finally conclusions are derived in section VI.

\section{RELATED WORK}

Most of the work in WMSN is related to multimedia sensor hardware [3][4]. S. Soro et al. [6] analyze how coverage-based algorithms designed for scalar sensors do not behave well in a video sensor network in terms of environment monitoring. The reason is the specific situation of sensing region (i.e., FoV) of a multimedia node with respect to the node location. In [8], the authors propose an optimal polynomial time algorithm for computing the worst-case breach coverage in FoV sensor networks, where "breach" is defined as the closest distance of the moving target to any sensor. The algorithm uses FoV-Voronoi graphs, only FoV shared areas participate in the construction of the Voronoi graph. The algorithm is used to inspect the probability that a random deployment results in an unobserved path between initial and final observed regions. The authors of [7] present an algorithm that enables self configurable sensor orientation calculation. The goal is finding that camera orientation that minimizes occlusion viewpoints and overlapping areas.

Clustering has been studied in great depth in the field of wireless scalar sensor networks. The work [5] surveys present clustering protocols and algorithms in this field and provide the main keys for designing such algorithms. Cluster formation and cluster-head selection manners are the key factors in clustering algorithms. Most of the clustering algorithms form clusters based on sensor neighborhood (radiocoverage) or on distance/radii from the cluster-head. The number of clusters and cluster-size are parameters that usually impact cluster formation procedures. K. Obrackza et al. [9] state the fact that directional FoV should be the key parameter to form clusters in WMSN and also highlight examples in which video sensor spatial-based collaboration provides robust object detection by cross-validating information.

On the other hand some algebra and geometric calculations are related to our research. Works [10][11][12][13] present different methods for testing whether two triangles intersect each other. Their techniques solve the basic sets of linear equations associated with the problem and exploit the relations between these sets to distinguish the existing overlaps.

\section{Node Clustering FOR WMSNS}

Multimedia sensors, such as cameras, are powerful multidimensional sensors that can capture a directional view. We assume wireless sensor nodes with fixed lenses providing a $\theta$ angle FoV, densely deployed in a random manner. The assumption of fixed lenses is based on the current WMSN platforms. Almost all of them (Cyclops, MicrelEye, CITRIC, Panoptes, Meerkats [14][15][16][17][18]) have fixed lenses and only high powered PTZ cameras have movement capabilities. We consider a monitor area with $\mathrm{N}$ wireless multimedia sensors, represented by the set $S=\left\{S_{1}, S_{2}, \ldots, S_{N}\right\}$ randomly deployed. Each sensor node is equipped to learn its location coordinates and orientation information via any lightweight localization technique for wireless sensor networks. Without loss of generality, let us assume that nodes in the set $\mathrm{S}$ belong to a single-tier network or the same tier of a multitier architecture. The following definitions will be used throughout the paper:

- Field of View (FoV): refers to the directional view (see Fig. 1) of a multimedia sensor and it is assumed to be an isosceles triangle (two-dimensional approximation) with vertex angle $\theta$, length of congruent sides $R_{s}$ (sensing range) of the sensor and orientation $\alpha$. The sensor is located at point $\mathrm{A}\left(\mathrm{x}_{\mathrm{A}}, \mathrm{y}_{\mathrm{A}}\right)$.

- Cluster $\left(C_{j}, j=1, \ldots, M\right)$ : consists of a subset of multimedia nodes with high overlapping FoV areas. The size of overlapping area between FoVs of two nodes determines whether they can be in the same cluster.

- Clustering threshold $(\gamma)$ : defines the minimum percentage of node's FoV area that is required to be overlapped for membership in a cluster.

\section{A. Overlapping areas between FoV of multimedia nodes}

It is obvious that there is no overlap between FoV of two nodes if the Euclidean distance between them is more than $2 \mathrm{R}_{\mathrm{s}}$. Otherwise, it is possible to have overlapped regions between their FoV depending on the orientation angles $\alpha$. For calculating the FoV overlapping area of two nodes, first we survey intersection of the triangles that are the representatives of their FoVs. Second, if they intersect each other, we find the intersection polygon and at last, compute the area of the polygon.

For this purpose, in the first step, we define the equations of sides of each triangle using coordinates of vertices of each triangle. The coordinates of the main vertex A (Fig. 1) is known according to the location of node in the space and the coordinates of vertex $\mathrm{P}_{1}$ and $\mathrm{Q}_{1}$ are:

$$
\begin{gathered}
\mathrm{x}_{\mathrm{P}_{1}}=\mathrm{x}_{\mathrm{A}}+\mathrm{R}_{\mathrm{s}} \cdot \cos (\alpha) \\
\mathrm{y}_{\mathrm{P}_{1}}=\mathrm{y}_{\mathrm{A}}+\mathrm{R}_{\mathrm{s}} \cdot \sin (\alpha) \\
\mathrm{x}_{\mathrm{Q}_{1}}=\mathrm{x}_{\mathrm{A}}+\mathrm{R}_{\mathrm{s}} \cdot \cos ((\alpha+\theta) \bmod 2 \pi) \\
\mathrm{y}_{\mathrm{Q}_{1}}=\mathrm{y}_{\mathrm{A}}+\mathrm{R}_{\mathrm{s}} \cdot \sin ((\alpha+\theta) \bmod 2 \pi)
\end{gathered}
$$

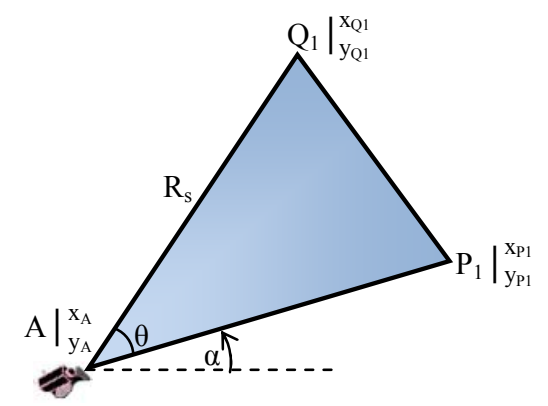

Figure 1. FoV of a multimedia sensor 
Using the coordinates of $\mathrm{A}, \mathrm{P}_{1}$ and $\mathrm{Q}_{1}$, we can define the equation of lines as representatives of three sides of the FoV triangle.

$$
\begin{gathered}
\overline{\mathrm{P}_{1} \mathrm{Q}_{1}}: y-\mathrm{y}_{\mathrm{P}_{1}}=\frac{\mathrm{y}_{\mathrm{Q}_{1}}-\mathrm{y}_{\mathrm{P}_{1}}}{\mathrm{x}_{\mathrm{Q}_{1}}-\mathrm{x}_{\mathrm{P}_{1}}} \cdot\left(\mathrm{x}-\mathrm{x}_{\mathrm{P}_{1}}\right) \\
\overline{\mathrm{AP}_{1}}: \mathrm{y}-\mathrm{y}_{\mathrm{A}}=\left(\mathrm{x}-\mathrm{x}_{\mathrm{A}}\right) \cdot \tan (\alpha) \\
\overline{\mathrm{AQ}_{1}}: \mathrm{y}-\mathrm{y}_{\mathrm{A}}=\left(\mathrm{x}-\mathrm{x}_{\mathrm{A}}\right) \cdot \tan ((\alpha+\theta) \bmod 2 \pi)
\end{gathered}
$$

In second step, we survey the intersection of each side of each triangle to all sides of the other triangle (i.e., the perimeter of the triangle). Furthermore, in the case of intersection, the vertices and also the sides of the overlapped polygon and order of them are found in this step. An example of Intersection polygon of two FoV belonging to nodes $A=\left(x_{A}\right.$, $\left.\mathrm{y}_{\mathrm{A}}\right)$ with the orientation $\alpha_{1}$ and $\mathrm{B}=\left(\mathrm{x}_{\mathrm{B}}, \mathrm{y}_{\mathrm{B}}\right)$ with the orientation $\alpha_{2}$ is illustrated in Fig. 2.

An intersection point $\mathrm{V}$ of two lines representing two sides of FoVs will be an acceptable vertex of the overlapped polygon, if it lies among the vertices associated with those two sides. As illustrated in Fig. 2, the line representing $\mathrm{AP}_{1}$ of FoV of node $\mathrm{A}$ intersects the line that represent $\mathrm{BP}_{2}$ of $\mathrm{FoV}$ related to node $\mathrm{B}$ in point $\mathrm{V}_{1} . \mathrm{V}_{1}$ can be considered as a vertex of the polygon because $V_{1}$ is located between $A$ and $P_{1}$ also between $\mathrm{B}$ and $\mathrm{P}_{2}$. The desired condition for a point $\mathrm{V}$ to be an acceptable intersection point is stated in (8). This subject is noticeable because any two anti-parallel lines obviously have an intersection point in a two dimensional space. On the other hand, each side of a FoV is a segment of a line. An instance of non-acceptable intersection points is illustrated in Fig. 3.a.

The intersection of each side of one triangle with all sides of another triangle consists of at most two points. Fig. 2 shows the case in which each side of each triangle intersects the perimeter of other one exactly in two points $V_{i}$ and $V_{j}$, becoming the segment $\mathrm{V}_{\mathrm{i}} \mathrm{V}_{\mathrm{j}}$ one of the sides of the intersection polygon. However, there are other situations in which the intersection of one side of a triangle with the perimeter of another triangle occurs only at one point, resulting in that one of the vertices associated with that side lies within the second triangle and will become one of the vertices of the polygon (Fig. 3.b and 3.c).

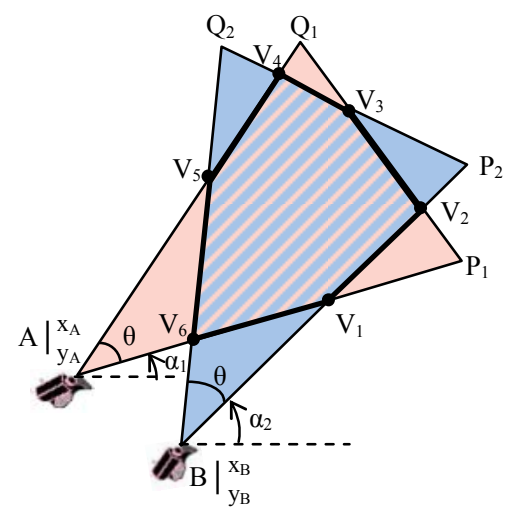

Figure 2. An example of intersection polygon of two FoVs

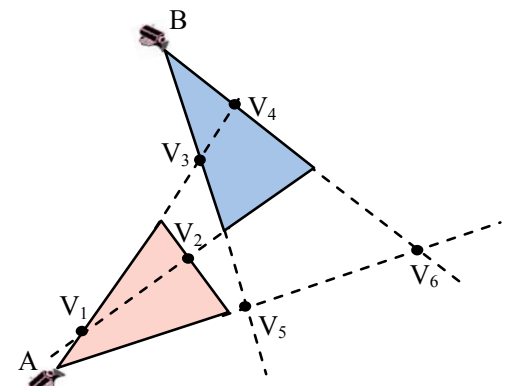

(a)

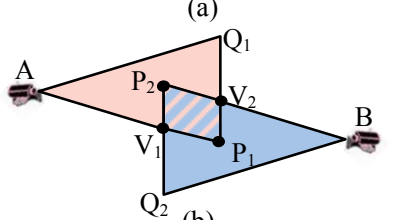

(b)

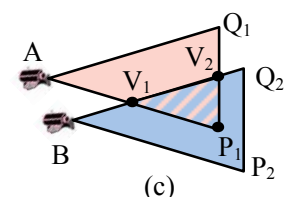

Figure 3. Examples: a) non-acceptable intersection points, b) and c) side intersection in only one point

$$
\begin{aligned}
& \left(\left(\mathrm{x}_{\mathrm{A}} \leq \mathrm{x}_{\mathrm{V}} \leq \mathrm{x}_{\mathrm{P}_{1}}\right) \wedge\left(\mathrm{x}_{\mathrm{B}} \leq \mathrm{x}_{\mathrm{V}} \leq \mathrm{x}_{\mathrm{P}_{2}}\right)\right) \vee \\
& \left(\left(\mathrm{x}_{\mathrm{A}} \leq \mathrm{x}_{\mathrm{V}} \leq \mathrm{x}_{\mathrm{P}_{1}}\right) \wedge\left(\mathrm{x}_{\mathrm{B}} \geq \mathrm{x}_{\mathrm{V}} \geq \mathrm{x}_{\mathrm{P}_{2}}\right)\right) \vee \\
& \left(\left(\mathrm{x}_{\mathrm{A}} \geq \mathrm{x}_{\mathrm{V}} \geq \mathrm{x}_{\mathrm{P}_{1}}\right) \wedge\left(\mathrm{x}_{\mathrm{B}} \geq \mathrm{x}_{\mathrm{V}} \geq \mathrm{x}_{\mathrm{P}_{2}}\right)\right) \vee \\
& \left(\left(\mathrm{x}_{\mathrm{A}} \geq \mathrm{x}_{\mathrm{V}} \geq \mathrm{x}_{\mathrm{P}_{1}}\right) \wedge\left(\mathrm{x}_{\mathrm{B}} \leq \mathrm{x}_{\mathrm{V}} \leq \mathrm{x}_{\mathrm{P}_{2}}\right)\right)
\end{aligned}
$$

Finally, a decomposition method is used for calculating the area of the overlapped polygon in a 2D-plane [19] in third step. Let a polygon (W) be defined by its ordered vertices $\mathrm{V}_{\mathrm{i}}=\left(\mathrm{x}_{\mathrm{i}}, \mathrm{y}_{\mathrm{i}}\right)$ for $\mathrm{i}=0, \ldots, \mathrm{n}$ with $\mathrm{V}_{\mathrm{n}}=\mathrm{V}_{0}$. Also, let $\mathrm{P}$ be a reference point in the 2D-plane. For each edge $\mathrm{V}_{\mathrm{i}} \mathrm{V}_{\mathrm{i}+1}$ of the polygon $\mathrm{W}$, form the triangle $\Delta_{\mathrm{i}}=\mathrm{PV}_{\mathrm{i}} \mathrm{V}_{\mathrm{i}+1}$. Then, the area of the polygon $\mathrm{W}$ is equal to the sum of the signed areas of all the triangles $\Delta_{\mathrm{i}}$ for $i=0, \ldots, n-1$ :

$$
\mathrm{A}(\mathrm{W})=\sum_{\mathrm{i}=0}^{\mathrm{n}-1} \mathrm{~A}\left(\Delta_{\mathrm{i}}\right) \quad \text { where } \Delta_{\mathrm{i}}=\mathrm{PV}_{\mathrm{i}} \mathrm{V}_{\mathrm{i}+1}
$$

We refer to [19] for a detailed description of the method.

\section{B. Cluster formation and cluster membership}

Now, consider the set $\mathrm{S}=\left\{\mathrm{S}_{1}, \mathrm{~S}_{2}, \ldots, \mathrm{S}_{\mathrm{N}}\right\}$ of wireless multimedia nodes belonging to the same tier of a network randomly deployed. Cluster formation can be performed locally distributed at each sensor node or can be performed in a centralized manner at the sink. The proposed algorithm can work in both architectures and is only executed at node deployment.

We consider that cluster formation is executed in a centralized manner. The key factors in choosing central architecture are that: (i) For a distributed architecture each node should notify to the rest of the nodes about its location $A_{i}$ and its orientation $\alpha_{i}(i=1, \ldots, N)$. In a centralized architecture the nodes should notify to the sink their location and orientation. Note that this notification is only necessary at 
bootstrap phase. All phases of the clustering algorithm are executed only one time, right after node deployment. (ii) In many WSN applications, the sink has ample resources (storage, power supply, communication and computation) availability and capacity which make it suitable to play such a role. (iii) Collecting information by a sink node is more power efficient compared to spreading this information to each and every other node within the network. (iv) Having the global view of the network at the sink node facilitates provision algorithms for closer-to-optimal cluster determination; the global knowledge can be updated at the sink when new nodes are added or some nodes die. Such maintenance tasks can be regarded as a normal routine for the sink. (v) Finally, using a centralized scheme can relieve processing load from the sensors in the field and help in extending the overall network lifetime by reducing energy consumption at individual nodes.

The following phases are performed to establish and formation clusters:

Phase 1. Bootstrap: At node bootstrap, each sensor $\left\{\mathrm{S}_{\mathrm{i}}\right.$, $\mathrm{i}=1, \ldots, \mathrm{N}\}$ transmits its position $\left(\mathrm{x}_{\mathrm{i}}, \mathrm{y}_{\mathrm{i}}\right)$ and orientation $\alpha_{\mathrm{i}}$ to the sink. If there is a un-connected node in the net-work, it can not announce itself and thus will not be considered in the algorithm.

Phase 2. Clustering Formation: (i) Initially, the sink creates an empty cluster associated with an un-clustered multimedia node of S. So, that node will be clustered as the first member of the established cluster. (ii) Then, the sink finds the qualified un-clustered nodes for joining to that first member by computing the area of overlapped polygons of their FoV. From position and orientation of nodes, the sink computes the overlapped polygon area $\left(\mathrm{D}_{\mathrm{ij}}\right)$ between each un-clustered multimedia node and the first member of the established cluster as discussed in section II.A. If the computed overlapped area is equal or greater than the area determined by clustering threshold $(\gamma)$, the un-clustered node will be clustered as a member of the established cluster. (iii) When no more nodes can be added to the cluster, the sink takes a new un-clustered node, begins a new cluster and goes to step (ii). Fig. 4 shows the formation algorithm.

Phase 3. Membership notification: The sink notifies each first-member of clusters about its cluster-ID and members of the cluster.

Note that in a distributed architecture, the cluster formation algorithm would be the same at the cost of each sensor calculating which sensors have enough overlapping FoV with him.

\section{Formation Algorithm Test Results}

This section explains test results of cluster formation and membership approach. All sensor nodes have been configured with a FoV vertex angle $\theta=60^{\circ}$ and $R_{s}$ of $20 \mathrm{~m}$. A sensing field spanning an area of $120 \mathrm{~m} \times 120 \mathrm{~m}$ has been used. Sensor densities were varied to study the cluster formation from sparse to dense random deployments. Figures illustrate the average results of 50 independent running tests and the standard deviation (STD) of the results is specified in each graph. Each test corresponds to a new random deployment.

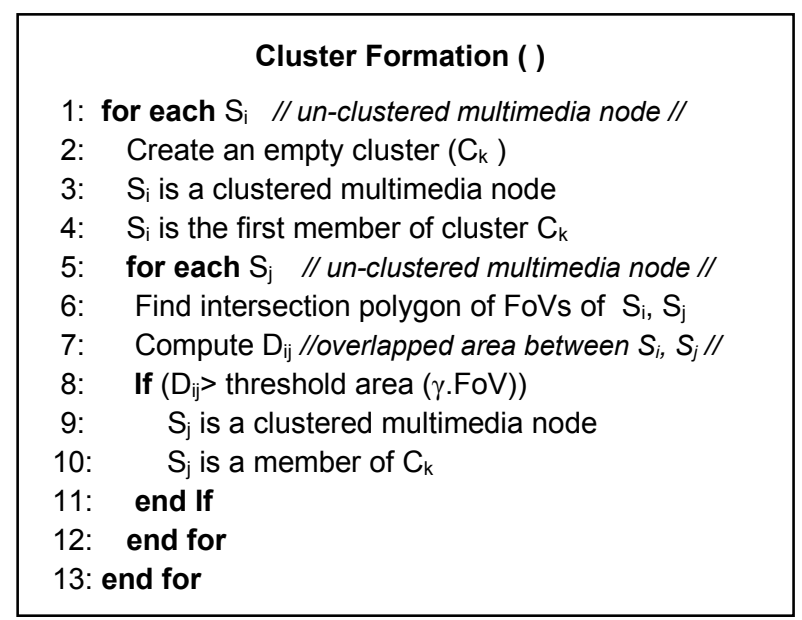

Figure 4. Cluster formation algorithm

Once a random deployment is defined, cluster-formation is obtained from node location, angle orientation and FoV, using the described algorithm that its complexity is $\mathrm{O}(\mathrm{N} \cdot \log \mathrm{N})$. Furthermore, as it was mentioned before, each node sends a packet to the sink in bootstrap phase and sink notifies each cluster (first member) via one packet consisting of membership results for that cluster (phase 3). Thus, the overhead of the algorithm is forwarding this number of packets ( $\mathrm{N}+\mathrm{Number}$ of clusters). Where $\mathrm{N}$ is the number of multimedia nodes, for number of clusters refer to Fig. 5.c.

\section{A. Average cluster-size and average number of clusters}

Fig. 5 shows the average of the three values: cluster size, maximum size and number of established clusters in the network with a clustering threshold $\gamma=0.5$. Increasing node density not only causes increment in number of clusters but also yields more overlapping areas among FoVs and raises cluster-size. Sparse networks have low average cluster-size because sparse deployments yield low overlapping sensing thus there will be low potential for node coordination. Nevertheless, dense wireless multimedia sensor networks can particularly benefit from node coordination where with a density of 300 nodes that covers $95 \%$ of the sensing area, the average cluster-size is of 2.67 and the average of maximum cluster-sizes comes to 7.57 .

\section{B. Effect of clustering threshold}

Clustering threshold determines the minimum region that is required to be overlapped between FoV of each node belonging to a given cluster and the FoV of the first member of that cluster. Fig. 6 shows the average cluster-size as a function of clustering threshold with for 300 nodes in the tier/network ( $95 \%$ coverage). Higher thresholds obviously restrict node memberships and decrease cluster- size, while lower thresholds increase the area covered by a given cluster at the cost of complexity of coordination. As an instance, in object tracking application, clusters having wider sensing area (lower clustering threshold) are more desired and reliable because the moving object lies in the domain of the same cluster for a longer time after detection by the cluster. 


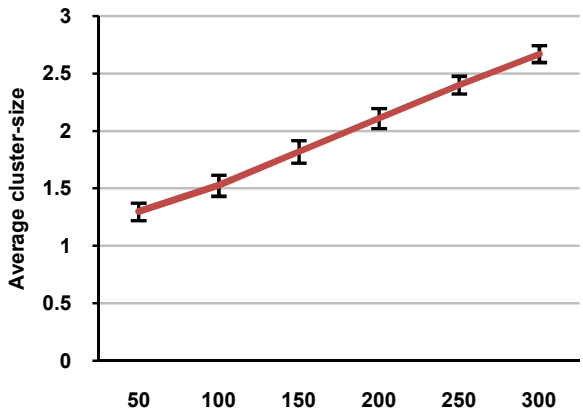

(a)

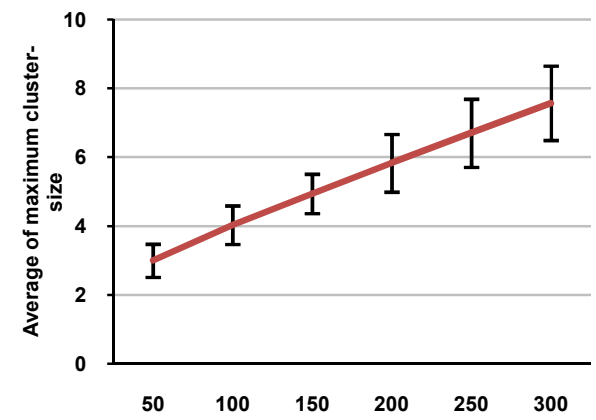

(b)

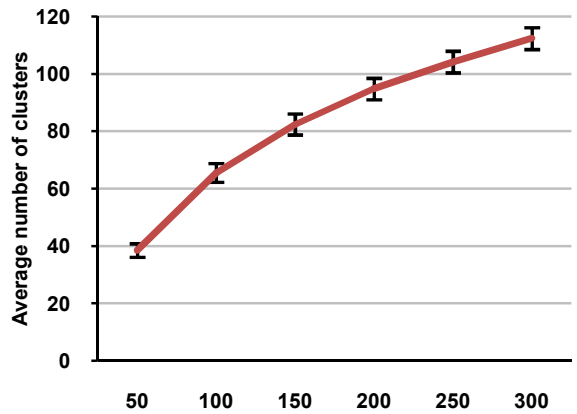

(c)

Figure 5. Average of a) cluster-size, b) maximum cluster-size, c) number of clusters for different node densities

\section{ENERGY CONSERVATION DEVELOPMENT}

In random deployed networks, the existence of obstacles produces a reduction of the sensing area because of FoV occlusion effect. So employing dense networks of low-cost, low-resolution and low-power multimedia sensor nodes instead of sparse networks of high-power, high-resolution sensors (e.g., PTZ) will be more beneficial. However, denser random deployments also cause having more overlapped areas among node FoVs [7]. The consequence is that nodes associated with overlapped FoVs mainly sense the same regions and will have similar views of the monitored area. Thus, a considerable amount of energy is wasted because of redundant sensing and processing in un-coordinated nodes. So, clustering nodes having high overlapped FoV can be the base for creating coordination among nodes (applying scheduling, turning jobs) within clusters to perform applications avoiding redundant sensing and processing and thus increase the lifetime and/or efficiency particularly in dense networks. However, we note that clustering also allows multi-view monitoring ability at the cost of overlapping nodes monitoring the same area/object concurrently without energy savings.

As an instance and with the aim of discussing multimedia sensor coordination benefits, we assume a random deployment tier/network in which nodes periodically are awakened, capture an image, process/send the image to sink and sleep (e.g., SensEye [20]). While keeping the cameras always on achieves low-latency detection, it is very energy-inefficient, since cameras and nodes will continuously consume energy. Thus, the cameras and the sensor nodes are duty-cycled and awakened periodically to monitor the environment. Using the

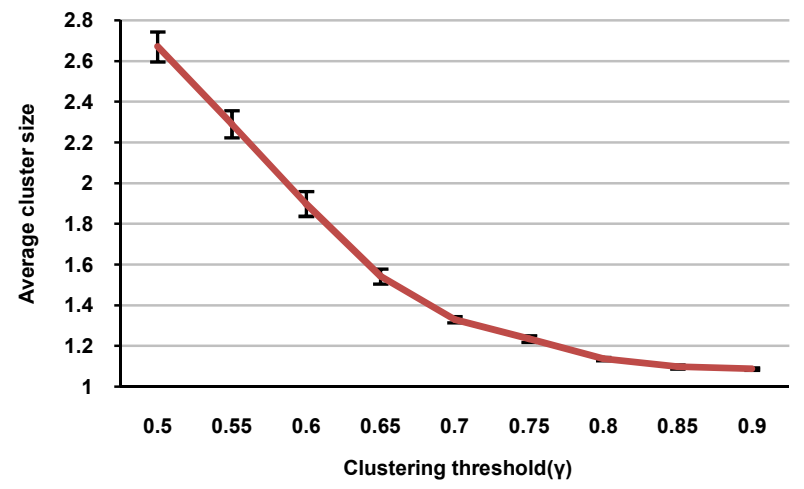

Figure 6. Average cluster-size as a function of clustering threshold clustering scheme, we can divide the environment sensing task in clusters of overlapping FoVs. Each cluster covers a region by its members with a high degree of overlapping; as regards an event can happen in each of these regions, all clusters sense their domains concurrently by their members in a collaborative manner. In each cluster, the first member is programmed to sequentially awaken members to capture an image, process or send image and sleep, in an intermittent way. Each node belonging to a given cluster is involved in image capturing from its unique perspective in its intermittence. In this way, members of the cluster participate in multimedia tasks sequentially by time intervals. Fig. 5 showed that average cluster size may be between 2 and 2.6 for node densities in the range of 150 to 300 and maximum cluster size is between 5 and 7.5. These nodes can save energy coordinating in performing the multimedia task because they sense an overlapping area. To evaluate what is the outcome of this kind of coordination, let us define as $\mathrm{N}$ the number of nodes in the mentioned tier that without coordination periodically sense their environment with the period of time $\mathrm{T}$. The energy used to sense the area is given by the energy consumed by the $\mathrm{N}$ nodes during this period of time while in the clustered scheme, the number of nodes that are activated with the same period of time is equal to the number of clusters, because each cluster employs one of its members in each interval and keeps the other nodes in sleep mode until next interval. Therefore, the consumed energy during period $\mathrm{T}$ is reduced compared to the energy consumed by $\mathrm{N}$ nodes without this coordination. We define the Energy Conservation Ratio (ECR) for each cluster as the ratio of the total amount of energy consumed by nodes belonging to the cluster during each interval $(\mathrm{T})$ in two un-clustered and clustered cases for multimedia applications. Equation (10) indicates the consumed energy during the interval $\mathrm{T}$ by a given node that is awakened:

$$
\mathrm{E}=\left(\mathrm{T}_{\text {sleep }} \cdot \mathrm{P}_{\text {sleep }}+\left(\mathrm{E}_{\mathrm{w}_{-} \text {up }}+\mathrm{E}_{\text {cap }}+\mathrm{E}_{\text {process }}\right)\right)
$$

Where $\mathrm{T}_{\text {sleep }}$ and $\mathrm{P}_{\text {sleep }}$ are the period and power consumption for a node in sleep mode. $\mathrm{E}_{\mathrm{w}_{-} \text {up }}, \mathrm{E}_{\mathrm{cap}}$ and $\mathrm{E}_{\text {process }}$, respectively are the energies consumed for waking up a node, capturing a picture and performing desired task. As in each cluster one of the cluster members is activated in each interval and the other members are in sleep mode, the energy consumed by nodes belonging to the cluster (with cluster size: CS) during each interval is equal to:

$$
\mathrm{E}_{\text {cluster }}=\mathrm{E}+(\mathrm{CS}-1) \cdot \mathrm{P}_{\text {sleep }} \cdot \mathrm{T}
$$


Therefore, the ECR for the cluster is:

$$
\mathrm{ECR}=\frac{\mathrm{CS} \cdot \mathrm{E}}{\mathrm{E}_{\text {cluster }}}
$$

Obviously, clusters with more number of nodes conserve more energy and have higher ECR since an overlapped region is shared among more number of coordinated members. For a tier/network consisting of $\mathrm{N}$ multimedia nodes, we can define average ECR according to average of cluster size (AvgCS):

$$
\mathrm{ECR}_{\text {AVERAGE }}=\frac{\mathrm{AvgCS} \cdot \mathrm{E}}{\mathrm{E}+(\operatorname{AvgCS}-1) \cdot \mathrm{P}_{\text {sleep }} \cdot \mathrm{T}}
$$

Fig. 7 illustrates the average ECR in a network, employing Cyclops [14] as camera sensor embedded in MICA II, and T equal to 5 second and a $\gamma$ of 0.5 . we select object detection as the desired task for multimedia nodes. Dense deployments yield higher potential for energy conservation in nodes because of higher sizes of established clusters. As an example, in a network consisting of 300 nodes, the number of active nodes in each interval is the same as the number of clusters which is 112.36 in average (see Fig. 5.c). In this case, each cluster in average consists of 2.67 members while in each interval one of them is active and the others are saving their energy in sleep mode and thus the energy conservation ratio in the network rises to 2.14 .

\section{CONCLUSION}

In this paper, a clustering method for multimedia wireless sensor networks is proposed. Cluster-membership is decided based on FoV overlapping areas instead of neighborhood in contrast to scalar sensors. The main objectives in this work are to achieve ability of coordination among cluster nodes in sensing and processing tasks and also to develop energy conservation in the clustered multimedia nodes. The coordination among multimedia nodes can considerably prevent wasting power avoiding redundant sensing, processing or sending similar multimedia data. Thus, it prolongs network lifetime particularly in dense networks that are usually deployed with a high number of low power, low resolution and inexpensive multimedia nodes in random manner. The proposed algorithm can work in both centralized and distributed architectures and is only executed at node deployment. We select a centralized manner to perform it regarding power efficiency and enduring a negligible overhead for WMSNs.

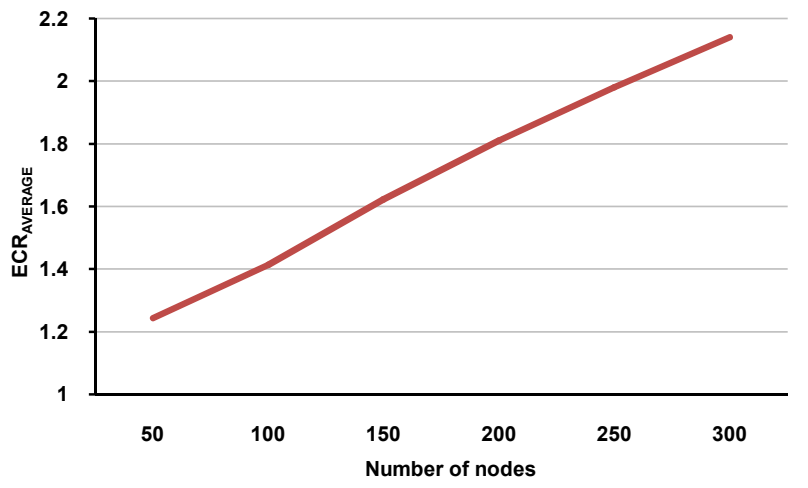

Figure 7. Average Energy Conservation Ratio for different node densities in a clustered tier/network with respect to the un-clustered ordinary network

\section{ACKNOWLEDGMENT}

Authors thank Spanish ministry of science and technology for providing facilitates to present this work.

\section{REFERENCES}

[1] F. Akyildiz, W. Su, Y. Sankarasubramaniam and E. Cayirci, "Wireless sensor networks: a survey," Computer Networks (Elsevier) Journal, Vol. 38, No. 4, pp. 393-422, March 2002.

[2] Y. Chong and S. P. Kumar "Sensor networks: evolution, opportunities, and challenges," Proceedings of the IEEE, vol. 91, pp. $1247-1256$, 2003.

[3] F. Akyildiz, T. Melodia, and K. R. Chowdhury, "A survey on wireless multimedia sensor networks," Computer Networks (Elsevier) Journal, Vol. 51, pp. 921-960, March 2007.

[4] I.F. Akyildiz, T. Melodia, K.R. Chowdhury, "Wireless multimedia sensor networks: applications and testbeds," Proceedings of the IEEE, Vol. 96, no. 10, pp. 1588 - 1605, October 2008.

[5] A. Abbasi, M. Younis, "A survey on clustering algorithms for wireless sensor networks," Computer Communications (Elsevier) journal, Vol. 30, Issue 14-15, pp. 2826-2841, June 2007.

[6] S. Soro, W. Heinzelman, "On the coverage problem in video-based wireless sensor networks," in Proceeding. of IEEE International Conference of Broadband Communications and Systems (BroadNets), Boston, MA, USA, October 2005.

[7] N. Tezcan, W. wang, "Self-orienting wireless multimedia sensor networks for occlusion-free viewpoints," Computer Networks (Elsevier) Journal, Vol 52, Issue 13, pp. 2558-2567, June 2008.

[8] J. Adriaens, S. Megerian, M. Potkonjak, "Optimal worst-case coverage of directional field-of-view sensor networks," Proceeding of IEEE SECON, 2006.

[9] K. Obraczka, R. Manduchi, J.J. Garcia-Luna-Aveces, "Managing the Information Flow in Visual Sensor Networks," Proceedings of the 5th International Symposium on Wireless Personal Multimedia Communications, October 2002.

[10] O. Tropp, A. Tal, L. Shimshoni, "A fast triangle to triangle intersection test for collision detection," Computer Animation Virtual Worlds, Vol. 17, pp. 527-535, 2006.

[11] H. Shen, P. A. Heng, Z. Tang, "A fast triangle-triangle overlap test using signed distances," Journal of Graphics Tools, Vol. 8, No. 1, pp. 17-24, 2003.

[12] P. Guigue, O. Devillers, "Fast and robust triangle-triangle overlap test using orientation predicates," Journal of Graphics Tools, Vol. 8, No. 1, pp. 25-42, 2003.

[13] T, Moller, "A fast triangle-triangle intersection test," Journal of Graphics Tools, Vol. 2, No. 2, pp. 25-30, 1997.

[14] M. Rahimi, R. Baer, O. I. Iroezi, J. C. Garcia, J. Warrior, D. Estrin, M. Srivastava, "Cyclops: in situ image sensing and interpretation in wireless sensor networks," in Proc. ACM Conference on Embedded Networked Sensor Systems, pp.192 - 204, 2005.

[15] Kerhet, M. Magno, F. Leonardi, A. Boni, and L. Benini, "A low-power wireless video sensor node for distributed object detection," Journal of Real-Time Image Processing, vol. 2, pp. 331-342, 2007.

[16] P. Chen, P. Ahammed, C. Boyer, S. Huang, L. Lin, E. Lobaton, M. Meingast, S. Oh, S. Wang, P. Yan, A. Y. Yang, C. Yeo, L. C. Chang, D. Tygar, and S. S. Sastry, "CITRIC: a low-bandwidth wireless camera network platform," in Proc. International Conference on Distributed Smart Cameras, pp. 1-10, 2008.

[17] W. C. Feng, E. Kaiser, M. Shea, W. C. Feng, L. Baillif, "Panoptes: scalable low-power video sensor networking technologies," Transactions on Multimedia Computing, Communications and Applications, vol. 1, pp. 151-167, May 2005.

[18] B. Margi, X. Lu, G. Zhang, G. Stanek, R. Manduchi,and K. Obraczka, "Meerkats: A power-aware, self-managing wireless camera network for wide area monitoring," in Distributed Smart Cameras Workshop SenSys06, 2006.

[19] http://softsurfer.com/

[20] P. Kulkarni, D. Ganesan, P. Shenoy, and Q. Lu, "SensEye: a multi tier camera sensor network," in Proc. ACM International Conference on Multimedia, pp. $229-238,2005$. 